\title{
Yeast ribonucleic acid: Effects on learned behavior in the rat'
}

ALLAN R. WAGNER, J. BROOKS CARDER AND WILLIAM W. BEATTY ${ }^{2}$

YALE UNIVERSITY

Rats chronically treated with yeast ribonucleic acid showed enhanced acquisition of an escape response, thus replicating a previously reported effect. The nature of the enhancement, however, as well as the results from a food-rewarded discrimination task, does not suggest that yeast ribonucleic acid improves the learning or memory processes in the rat.

There are few studies in which learning or memory in animals has reportedly been enhanced by a pharmacological agent. A recent investigation by Cook, Davidson, Davis, Green, \& Fellows (1963) employing yeast ribonucleic acid, has received considerable attention, not only because it apparently illustrates such an enhancement, but also because of the implications it may have for recent theories (e.g. Hyden, 1964; Gaito, 1963; Landauer, 1964) concerning the role of nucleic acids in experiential memory.

Cook et al compared the escape behavior of rats who had received 28 or 53 daily, intraperitoneal injections of yeast RNA, with that of saline-treated control animals. A conditioning trial involved placing $S$ on a grid floor and then simultaneously presenting a buzzer and an elertric shock through the floor. The stimuli terminated on such trials after $30 \mathrm{sec}$. or when S responded by climbing a vertical pole suspended above the center of the floor. RNA injected Ss took fewer trials to adopt a consistent pole-climbing response to the shock, and evidenced shorter response latencies than control Ss. Furthermore, RNA-treated Ss were more likely to respond on trials when the buzzer was presented alone, either when interspersed with shockbuzzer trials or during a block of such (extinction) trials.

The present experiments were designed to further clarify the effects of yeast RNA on learned behavior in the rat.

\section{Experiment 1}

An attempt was made to replicate the Cook et al experiment under as identical conditions (Cook \& Weidley, 1957; Cook, Davidson, Davis, Green, \& Fellows, 1963) as possible. Initial work indicated, however, that in our apparatus, with the same nominal shock intensity employed by Cook et al (.8 ma), all Ss, regardless of their injection history, acquired the response so rapidly that there was little opportunity to detect any possible drug effects. Consequently, a somewhat lower nominal shock intensity $(.5 \mathrm{ma})$ was employed in the study reported.

Male albino rats were obtained from the Charles River Breeding Laboratory and from 70 days of age were treated daily with intraperitoneal injections of either yeast RNA (160 mg/kg in a $100 \mathrm{mg} / \mathrm{ml}$ solution) or equal volumes of physiological saline. All behavioral observations were begun after at least 30 days of injections. Five RNA and five saline, control Ss were given 10 training trials each day for four days. In addition, the buzzer was presented alone without shock on six test trials interspersed among the last 30 trials.

All five Ss in the RNA group made the pole-climbing response on every conditioning or test trial after trial 4. In comparison, none of the control Ss made the response on a single trial. This finding confirms the observation of Cook et al that yeast RNA injections may facilitate the acquisition of the pole-climbing escape response. It does so, however, without necessarily implicating any improvement in the rats' learning or memory processes.

The control Ss never made the desired response, hence they never experienced the cessation of shock consequent upon this response. Each of the RNA injected $\mathrm{Ss}$, on the other hand, did make a pole-climbing response during their first several trials and subsequently profited from the experience by responding more consistently and more quickly on later trials. In the present experiment it is clear that RNA injections increased the original likelihood of the to-be-rewarded response. If RNA had a similar effect in the Cook et al study, in which all Ss eventually learned, the differential acquisition rates which were observed would be most parsimoniously attributed to the interaction of similar learning and memory capacities with the different experiences, rather than appealing to different learning or memory capacities as well.

\section{Experiment II}

Two questions must apparently be considered concerning the action of yeast RNA: Why does it increase the original likelihood of pole-climbing in response to shock, and, does it, beyond this effect, also influence the learning or memory processes in a direct way?

A minimal expectation, granting the latter possibility, would be that yeast RNA treatment would benefit the acquisition of a variety of different responses involving a variety of motivation-reward conditions. A second experiment suggests that however general the effects of yeast RNA may be, they do not appear to extend to the acquisition of food-rewarded discrimination behavior.

Fourteen RNA and 14 saline-control rats were injected according to the regime described in Experiment I. They were also placed on a limited feeding schedule 
adjusted to maintain their weights between 70 and 80 percent of the mean concurrent weight of saline Ss on an ad lib diet. The two groups were then trained on a food-rewarded discrimination task.

The apparatus was a $\mathrm{Y}$-maze, the dimensions of which have been described elsewhere (Wagner \& Miller, 1962), consisting of a grey stem, and one black and one white arm. On each trial $\mathrm{S}$ was placed in the stem of the maze and rewarded with six $.045 \mathrm{gm}$ pellets if it entered an arm of one brightness and nonrewarded if it entered the alternate arm. Guillotine doors at the choice point were used to prevent retracing once an arm was entered or to block entrance to one arm on forced trials. Half of the Ss in each group were trained to go to the black arm and half to the white. For any $S$ the two arms were interchanged so that each brightness was located equally as often on the left as on the right.

After habituation to the maze, the groups received 11 daily training sessions of eight trials each, patterned after a procedure employed by Spence, Goodrich, \& Ross (1959). In each block of four trials the first trial allowed a choice between the two arms, while on each of the next three one of the two arms was blocked so as to equate the total number of exposures to the two brightnesses for each animal.

The major data were the preference behaviors on the two choice trials in each session, but running speeds were also measured on the forced trials to the two discriminanda. Figure 1 presents the mean percentage choice of the rewarded arm for the two groups on successive training days. As may be seen, there was no advantage for the RNA group in the acquisition of this

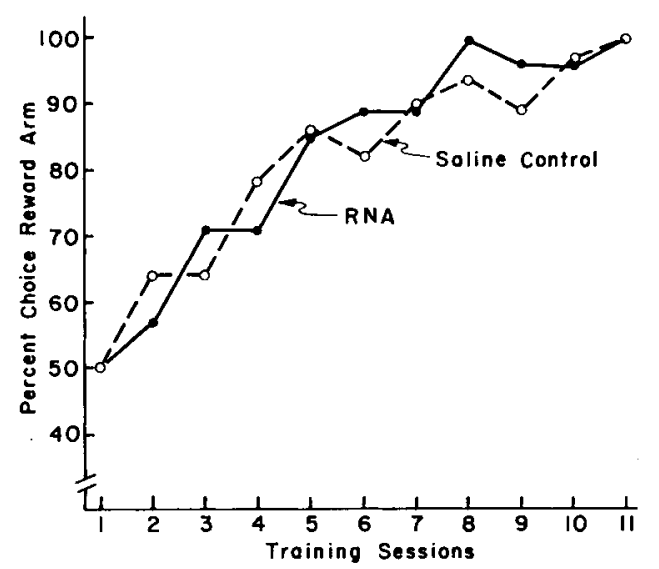

Fig. 1. Percent choice of reward arm in a brightness discrimination for groups of rats receiving daily injections of yeast RNA or physiological saline. discrimination. The only notable difference in the behavior of the two groups in this experiment was a tendency for the running speeds of the RNA group to be somewhat faster to both the rewarded and nonrewarded discriminanda than those of the saline group, but this difference was not statistically reliable, $\mathrm{F}=2.64$, $\mathrm{df}=$ $1 / 26, p>.10$.

\section{Discussion}

From these studies it would appear that although yeast RNA may influence rats' performances in some learning situations, it is doubtful that it does so through any direct effect upon the learning or memory process.

Since RNA was found to increase the original likelihood of responding in a shock situation but not to influence choice behavior in a food reward task, it is possible that it works to sensitize $S$ to shock. In a companion experiment, however, in which the threshold value of shock was determined for producing movement in a stabilimeter, no difference was observed between the means of $18 \mathrm{RNA}-$ and 14 saline-injected Ss.

Another interpretation, consistent, not only with the results of Experiment I, but also with the tendency for the RNA Ss in Experiment II to run somewhat faster than their controls, is that RNA increases general activity level. Again, however, an assessment of this possibility in an activity wheel produced no difference between the means of 18 RNA- and 18 saline-injected Ss

In summary, the effect of yeast RNA as originally reported by Cook et al, seems likely to result from al increased untrained likelihood of pole-climbing in re. sponse to shock, but the basis of this increased likeli. hood remains obscure.

\section{Reterences}

Cook, L., Davidson, A. B., Davis, D. J., Green, H., \& Fellows E. J. Ribonucleic acid: effect on conditioned behavior in rats Science, 1963, 141, 268-269.

Cook, L., \& Weidley, E. Drug effects in escape conditioning. Ant N. Y. Acad. Sci., 1957, 66, 740 .

Gaito, J. DNA and RNA as memory molecules. Psychol. Rer. 1963, 70, 471-480.

Hyden, H. RNA - A functional characteristic of the neuron and it giia. In M. A. Brazier (Ed.), Brain function. Vol. II Berkeley University of Calif. Press, 1964. Pp. 29-68.

Landauer, T. K. Two hypotheses concerning the biochemical basi of memory. Psychol. Rev., 1964, 71, 167-179.

Spence, K. W., Goodrich, K. P., \& Ross, L. E. Performance in dif ferential conditioning and discrimination learning as a function of a hunger and relative response frequency. $J$. exp. Psychol., $1959,58,8-161$

Wagner, A. R., \& Miller, N. Choice behavior and resistance to extinction. Psychol. Rev., 1962, 12, 105-108.

\section{Notes}

1. This research was supported in part by National Science Foundation Grant No. G-24015.

2. The junior authors are now at the University of Pennsylvania and the University of Wisconsin, respectively. 